\title{
First Dark Matter Limits from a Large-Mass, Low-Background, Superheated Droplet Detector
}

\author{
J. I. Collar, ${ }^{1,2, *}$ J. Puibasset, ${ }^{1}$ T. A. Girard, ${ }^{3}$ D. Limagne, ${ }^{1}$ H. S. Miley, ${ }^{4}$ and G. Waysand ${ }^{1}$ \\ ${ }^{1}$ Groupe de Physique des Solides (UMR CNRS 75-88), Universités Paris $7 \&$ 6, 75251 Paris Cedex 05, France \\ ${ }^{2}$ CERN, EP Division, CH-1211 Geneve 23, Switzerland \\ ${ }^{3}$ Centro de Física Nuclear, Universidade de Lisboa, 1649-003 Lisbon, Portugal \\ ${ }^{4}$ Pacific Northwest National Laboratory, Richland, Washington 99352
}

(Received 28 January 2000)

\begin{abstract}
We report on the fabrication aspects and calibration of the first large active mass $(\sim 15 \mathrm{~g})$ modules of SIMPLE, a search for particle dark matter using superheated droplet detectors (SDDs). While still limited by the statistical uncertainty of the small data sample on hand, the first weeks of operation in the new underground laboratory of Rustrel-Pays d'Apt already provide a sensitivity to axially coupled weakly interacting massive particles (WIMPs) competitive with leading experiments, confirming SDDs as a convenient, low-cost alternative for WIMP detection.

PACS numbers: $95.35 .+\mathrm{d}$, 05.70.Fh, 29.40.-n
\end{abstract}

The rupture of metastability by radiation has been historically exploited as a method for particle detection. Perhaps its most successful application is the bubble chamber, where ionizing particles deposit enough local energy in a superheated liquid to produce vaporization along their wake. Apfel extended this concept in the form of superheated droplet detectors [1] (SDDs, aka bubble detectors), in which small drops (radius $\sim 10 \mu \mathrm{m}$ ) of the liquid are uniformly dispersed in a gel or viscoelastic medium. In a SDD the gel matrix isolates the fragile metastable system from vibrations and convection currents, while the smooth liquid-liquid interfaces impede the continuous triggering on surface impurities that occurs in bubble chambers. The lifetime of the superheated state is extended, allowing for new applications: SDDs are increasingly popular as neutron dosimeters, where the nucleated visible bubbles provide a reading of the radiation exposure. SIMPLE (superheated instrument for massive particle searches) aims to detect particle dark matter using SDDs. We report here on the sensitivity attained at the early prototype stage, already comparable to the best achieved with competing technologies.

In the moderately superheated industrial refrigerants used in SDDs, bubbles are produced only by particles having elevated stopping powers $(d E / d x \geqslant 200 \mathrm{keV} / \mu \mathrm{m})$ such as nuclear recoils. This is understood in the framework of the "thermal spike" model [2], common to bubble chambers: for the transition to occur, a vapor nucleus of radius $>r_{c}$ must be created, while only the energy deposited along a distance comparable to this critical radius $r_{c}$ is available for its formation. Hence, a double threshold is imposed: the deposited energy $E$ must be larger than the work of formation of the critical nucleus, $E_{c}$, and this energy must be lost over a distance $O\left(r_{c}\right)$, i.e., a minimum $d E / d x$ is required. More formally [3,4]

$$
\begin{aligned}
E>E_{c} & =4 \pi r_{c}^{2} \gamma / 3 \epsilon, \\
d E / d x & >E_{c} / a r_{c},
\end{aligned}
$$

where $r_{c}=2 \gamma / \Delta P, \gamma(T)$ is the surface tension, $\Delta P=$ $P_{V}-P, P_{V}(T)$ is the vapor pressure, $P$ and $T$ are, respectively, the operating pressure and temperature, $\epsilon$ varies in the range $[0.02,0.06]$ for different liquids [4,5], and $a(T) \sim O(1)[6]$.

Both thresholds can be tuned by changing the operating conditions: $\mathrm{keV}$ nuclear recoils like those expected from scattering of weakly interacting massive particles (WIMPs) (currently the favored galactic dark matter candidates [7]) are detectable at room $T$ and atmospheric $P$, allowing for a low-cost search free of the complications associated with cryogenic equipment. Most importantly, the threshold in $d E / d x$ provides an insensitivity to minimum-ionizing backgrounds that hamper the numerous WIMP detection efforts [8]. A mere $<10$ WIMP recoils $/ \mathrm{kg}$ target/day are expected and hence the importance of background reduction and/or rejection. SDDs of active mass $O(1) \mathrm{kg}$ can in principle considerably extend the present experimental sensitivity [9].

Prompted by the modest active mass of commercially available SDDs $(\sim 0.03 \mathrm{~g}$ refrigerant/dosimeter) and the need to control the fabrication process, we developed a 80 l, 60 bar pressure reactor dedicated to large-mass SDD production. It houses a variable-speed magnetic stirrer, heating and cooling elements, and micropumps for catalyst addition (we nevertheless favored thermally reversible food gels due to safety concerns in the handling of synthetic monomers). The fabrication of 11 SDD modules containing up to $3 \%$ in superheated liquid starts with the preparation of a suitable gel matrix; ingredients are selected and processed in order to avoid alpha emitters, the only internal radioemitters of concern [9]. A precise density matching between matrix and refrigerant is needed to obtain a uniform droplet dispersion, making water-based gels inadequate unless large fractions of inorganic salts are added, which can unbalance the chemistry of the composite and contribute an undesirable concentration of these contaminants [10]. We find that glycerol is for this and other 
reasons an additive of choice. It is purified using a bed of preeluted ion-exchanging resin specifically targeted at actinide removal. Polymer additives and gelating agent are washed in a resin bath. All components are forced through $0.2 \mu \mathrm{m}$ filters to remove motes that can act as nucleation centers. The resulting mixture is outgassed and maintained above its gelation temperature in the reactor. The refrigerant is distilled and incorporated to this solution at $P \gg$ $P_{V}(T)$ to avoid boiling during the ensuing vigorous stirring. After a homogenized dispersion $(r \sim 30 \pm 15 \mu \mathrm{m})$ of droplets is obtained, cooling, setting, and stepwise adiabatic decompression produce a delicate entanglement of superheated liquid and thermally reversible gel, the SDD. The detectors are refrigerated and pressurized during storage to inhibit their response to environmental neutrons.

SDDs can bypass the listed problems associated with a former [11] bubble chamber WIMP search proposal, but are not devoid of their own idiosyncrasies. For instance, the solubility of hydrogen-free refrigerant liquids in water-based gels is small (e.g., $0.002 \mathrm{~mol} / \mathrm{kg}$ bar for $\mathrm{R}-12, \mathrm{CCl}_{2} \mathrm{~F}_{2}$ ), yet sufficient to produce unchecked bubble growth via permeation after a few days of continuous SDD operation. The engorged bubbles lead to fractures, spurious nucleations, and depletion of the superheated liquid (commercial gel-based SDDs are designed for a few hours of exposure before recompression [12], a cycle that can be repeated a limited number of times). To achieve the long-term SDD stability needed for a WIMP search, we employ a multiple strategy: fracture formation can be delayed under a moderate $P \sim 2$ atm, or by choosing refrigerants with the lowest solubility in the matrix $\left(\sim 0.0003 \mathrm{~mol} / \mathrm{kg}\right.$ bar for $\left.\mathrm{R}-115, \mathrm{C}_{2} \mathrm{ClF}_{5}\right)$. Structuremaking inorganic salts produce a "salting-out" effect, i.e., further reduce the refrigerant solubility. Their use being inadvisable for the reasons above, we introduce instead polymers known to have a similar effect [13], such as polyvinylpyrrolidone (PVP). As a result of these measures, present SIMPLE modules are stable over $\sim 40 \mathrm{~d}$ of continuous exposure. Another example of SDD-specific problems is the formation of clathrate hydrates on droplet boundaries during fabrication or recompression. These metastable icelike structures are inclusions of refrigerant molecules into water cages [14] that shorten the lifetime of superheated drops encrusted by them via transfer mechanisms still not well understood [15]. Their presence may be responsible for a long-lived spurious nucleation rate observed in R-12 SDDs following fabrication [16]. This is addressed in SIMPLE with the addition of polymers such as PVCap or PVP, which act as kinetic inhibitors in their growth [14], and by use of large molecular size refrigerants like R-115, for which the formation of most hydrates is stoichiometrically forbidden $[14,15]$.

Prototype modules are tested in an underground gallery. The $27 \mathrm{~m}$ rock overburden and $\sim 30 \mathrm{~cm}$ paraffin shielding reduce the flux of muon-induced and cosmic fast neutrons, the main source of nucleations above ground. Inside the shielding, a water + glycol thermally regulated bath maintains $T$ constant to within $0.1{ }^{\circ} \mathrm{C}$. The characteristic violent sound pulse accompanying vaporization in superheated liquids $[17,18]$ is picked up by a small piezoelectric transducer in the interior of the module, amplified, and stored. Special precautions are taken against acoustic and seismic noise. Figure 1 displays the decrease in the spontaneous bubble nucleation rate brought by progressive purification of the modules.

The response of smaller SDDs to various neutron fields has been extensively studied $[6,19]$ and found to match theoretical expectations. However, large-size, opaque SDDs require independent calibration: acoustic detection of the explosion of the smallest or most distant droplets is not a priori guaranteed. The energy released as sound varies as $\left(P_{V}-P\right)^{3 / 2}$ [18], making these additional characterizations even more imperative for SDDs operated under $P>1 \mathrm{~atm}$. Two separate types of calibration have been performed to determine the target mass effectively monitored in SIMPLE modules and to check the calculation of the $T$-, $P$-dependent threshold energy $E_{\mathrm{thr}}$ above which WIMP recoils can induce nucleations [defined as the lowest energy meeting both conditions in Eq. (1) [9,20,21] ]. First, a liquid ${ }^{241} \mathrm{Am}$ source (an alpha emitter) is diluted into the matrix while still in the solution state. Following Eq. (1), the $5.5 \mathrm{MeV}$ alphas and $91 \mathrm{keV}$ recoiling ${ }^{237} \mathrm{~Np}$ daughters cannot induce nucleations at temperatures below $T_{\alpha}$ and $T_{\alpha r}$, respectively [9]. The expression $a=4.3\left(\rho_{v} / \rho_{l}\right)^{1 / 3}[6]$, where $\rho_{v}(T), \rho_{l}(T)$ are the vapor- and liquid-phase densities of the refrigerant, correctly predicts the observed $T_{\alpha}$ for both R-12 and R-115 at $P=1$ and $2 \mathrm{~atm}$. In the same conditions, the theoretical value of $\epsilon$ [22] for these liquids $(\epsilon \sim 0.026$, neglecting a small $T, P$ dependence) generates a good agreement with the experimental $T_{\alpha r}$ (Fig. 1 inset). Prior to extensive component purification, the spectrum in noncalibration

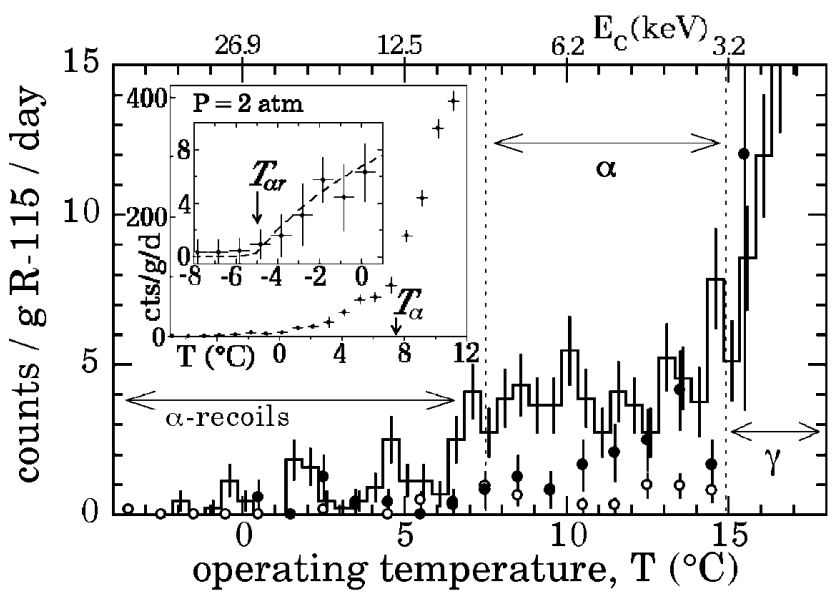

FIG. 1. SDD background at 90 mwe and $P=2$ atm, following cumulative steps of cleansing: histogram, double distillation of water and microfiltration; • , distillation of refrigerant and glycerin purification; $\circ$, gelatine and PVP purification. Inset: Rate induced in a calibration $\left(0.2 \mathrm{~Bq}{ }^{241} \mathrm{Am} / \mathrm{g}\right.$ gel $)$. The theoretical values of $T_{\alpha r}$ and $T_{\alpha}$ are indicated (the dashed line is a numerical simulation containing no free parameters). 
runs (Fig. 1 histogram) bears close resemblance to that produced by ${ }^{241} \mathrm{Am}$ spiking (Fig. 1 inset); the initial presence of a small $\left(\sim 10^{-4} \mathrm{pCi} / \mathrm{g}\right){ }^{228} \mathrm{Th}$ contamination, compatible with the observed rate, was confirmed via lowlevel alpha spectroscopy. Three regimes of background dominance are therefore delimited by vertical lines in Fig. 1: the sudden rise at $T \sim 15^{\circ} \mathrm{C}$ originates in high $d E / d x$ Auger electron cascades following interactions of environmental gammas with $\mathrm{Cl}$ atoms in the refrigerant [4,23]. The calculated $E_{c}$ for R-115 at $T=15.5^{\circ} \mathrm{C}$ and $P=2 \mathrm{~atm}$ is $2.9 \mathrm{keV}$, coincidental with the binding energy of $K$-shell electrons in $\mathrm{Cl}, 2.8 \mathrm{keV}$ (i.e., the maximum $E$ deposited via this mechanism). Thus, the onset of gamma sensitivity provides a welcome additional check of the threshold in the few $\mathrm{keV}$ region.

Alpha calibrations are not suitable for a rigorous determination of the overall sound detection efficiency because a large fraction of the added emitters drifts to gel-droplet boundaries during fabrication, an effect explained by the polarity of actinide complex ions [24] and dependent on matrix composition. While this migration does not affect $T_{\alpha}$ or $T_{\alpha r}$, it enhances the overall nucleation efficiency in a somewhat unpredictable manner [24]. To make up for this deficiency, SIMPLE modules have been exposed to a ${ }^{252} \mathrm{Cf}$ neutron source provided by the technical inspection and safety division (TIS/RP) at CERN. The resulting spectrum of neutron-induced fluorine recoils (Fig. 2 inset) mimics a typically expected one from WIMP interactions. A complete MCNP4a [25] simulation of the calibration setup takes into account the contribution from albedo and thermal neutrons. The expected nucleation rate as a function of $T$ is calculated as in $[9,19]$ : cross sections for the elastic, inelastic, $(n, \alpha)$, and $(n, p)$ channels of the refrigerant constituents are extracted from ENDFB-VI libraries. Look-up tables of the distribution of deposited energies as a function of neutron energy are built from the SPECTER code

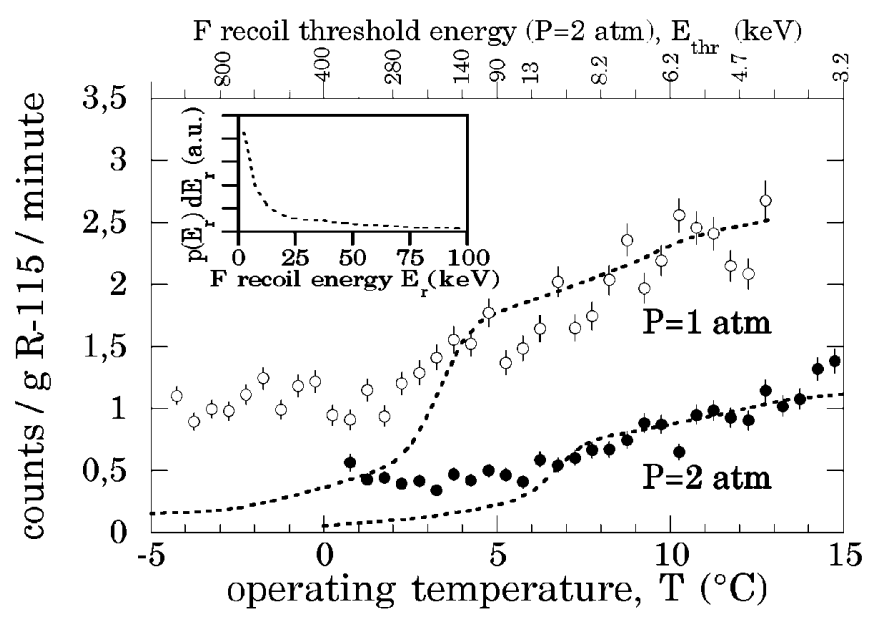

FIG. 2. ${ }^{252} \mathrm{Cf}$ neutron calibration of SIMPLE modules at the TIS/RP bench (CERN), compared with Monte Carlo expectations (dotted lines; see text). The signal-to-noise ratio was $>30$ at all times. Inset: Calculated energy spectrum of $F$ recoils during the irradiations.
[26]; stopping powers of the recoiling species are taken from SRIM98 [27]. Since $T$ was continuously ramped up during the irradiations at a relatively fast $1.1^{\circ} \mathrm{C} / \mathrm{hr}$, a small correction to it $\left(<1{ }^{\circ} \mathrm{C}\right)$ is numerically computed and applied to account for the slow thermalization of the module. Depending on $T$, the value of $E_{\mathrm{thr}}$ for elastic recoils in fluorine (the dominant nucleation mechanism in R-115) is set by either condition in Eq. (1), the other being always fulfilled for $E>E_{\text {thr }}[9,20]$. The handover from the second to the first condition at $T$ above $\sim 5.5^{\circ} \mathrm{C}\left(\sim 2.5^{\circ} \mathrm{C}\right)$ for $P=2 \mathrm{~atm}(P=1 \mathrm{~atm})$ is clearly observed in the data as two different regimes of nucleation rate (Fig. 2). A larger-than-expected response, already noticed in R-12 [6], is evident at low $T$ : the calculated $E_{\mathrm{thr}}$ there is too conservative (too high). This behavior appears well below the normal regime of SDD operation (which is at $T$ high enough to have $E_{\mathrm{thr}}=E_{c}$ ) and therefore does not interfere with neutron or WIMP detection. However, it is interesting in that it points at a higher than normal bubble nucleation efficiency from heavy particles, as discussed in early bubble chamber work [23]. A best fit to the overall normalization of the Monte Carlo over the full data set (Fig. 2, dotted lines) yields the fraction of refrigerant mass monitored with the present sound acquisition chain, 34\% $\pm 2 \%$ $(74 \% \pm 4 \%)$ of the total at $P=2 \mathrm{~atm}(P=1 \mathrm{~atm})$, a decisive datum to obtain dark matter limits.

The installation $500 \mathrm{~m}$ underground of modules identical in preparation and sound detection system to those utilized in ${ }^{252} \mathrm{Cf}$ calibrations started in July 1999. A decommissioned nuclear missile control center has been converted into an underground laboratory [28], facilitating this and other initiatives. The characteristics of this site (microphonic silence and unique electromagnetic shielding [28]) make it specially adequate for rare-event searches. Modules are placed inside a thermally regulated water bath, surrounded by three layers of sound and thermal insulation. A 7001 water neutron moderator, resting on a vibration absorber, completes the shielding. Events in the modules and in external microphones are time tagged, allowing to filter out the small fraction $(\sim 15 \%)$ of signals correlated to human activity in the immediate vicinity of the experiment. $P$ and $T$ are continually logged. The signal waveforms are digitally stored, but no event rejection based on pulse-shape considerations [10] is performed at this stage, eluding the criticisms [29] associated with some WIMP searches in which large data cuts are made.

The raw counting rate from the first SIMPLE module operated in these conditions appears in Fig. 3. Accounting for sound detection efficiency and a $62 \%$ fluorine mass fraction in R-115, limits can be extracted on the spindependent WIMP-proton cross section $\sigma_{W p}$ (Fig. 3). The cosmological parameters and method in [30] are used in the calculation of WIMP elastic scattering rates, which are then compared to the observed uncut nucleation rate at $T=10^{\circ} \mathrm{C}$ or $14^{\circ} \mathrm{C}$, depending on WIMP mass. The expected nucleation rate at $T$ [i.e., integrated for recoil energies $\left.>E_{\mathrm{thr}}(T)\right]$ from a candidate at the edge of the 


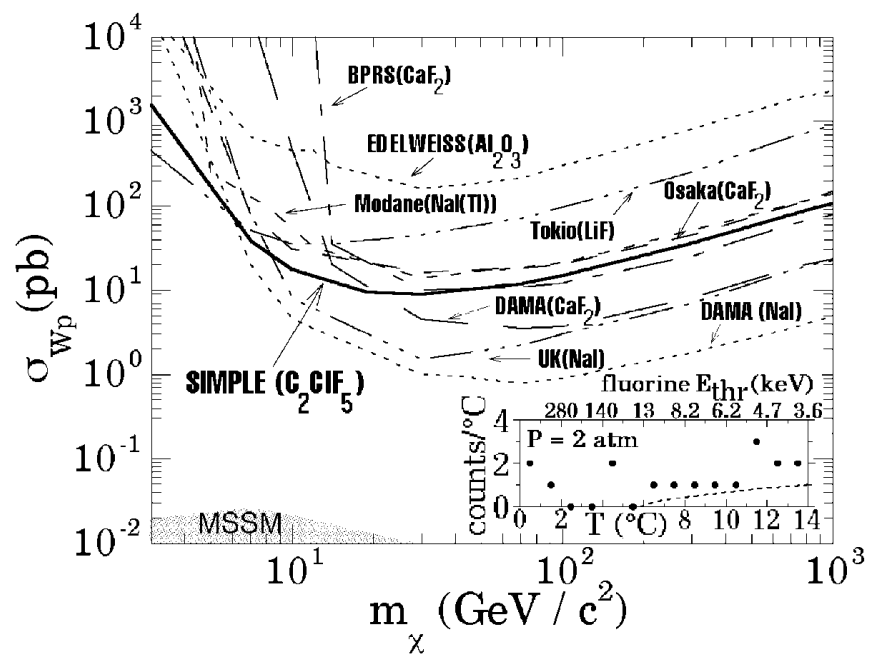

FIG. 3. $95 \%$ C.L. limits on $\sigma_{W p}$ extracted from $0.19 \mathrm{~kg} \mathrm{~d}$ of SDD exposure, compared with other experiments [8]. "MSSM" marks the tip of the region where a lightest supersymmetric partner is expected. Inset: Counting rate in the module $(9.2 \pm 0.1 \mathrm{~g}$ $\mathrm{R}-115, \Delta T=-0.75^{\circ} \mathrm{C} /$ day). The dotted line is the expected signal (corrected for $34 \%$ detection efficiency) from a WIMP of mass $m_{\chi}=10 \mathrm{GeV}$ and $\sigma_{W p}=5 \mathrm{pb}$ : present sensitivity is still limited by low statistics.

sensitivity of the leading DAMA experiment [8] $(\sim 1.5 \times$ $10^{4} \mathrm{kgd}$ of $\left.\mathrm{NaI}\right)$ is offered as a reference in Fig. 3: SIMPLE sensitivity is presently limited by the large statistical uncertainty associated with a short exposure, and not yet by background rate. A considerable improvement is expected after the ongoing expansion of the bath to accommodate up to 16 modules. In parallel to this, plastic module caps are being replaced by a sturdier design: runs using refrigerant-free modules show that a majority of the recorded events arise from pressure microleaks, correlated to the sense of $T$ ramping, able to stimulate the piezoelectric sensor. It must also be kept in mind that a $T$-independent, flat background implies a null WIMP signal, albeit this eventual approach to data analysis can only be exploited after a large reduction in statistical uncertainty is achieved.

The importance of the spin-dependent WIMP interaction channel (where $F$ is the optimal target [31]) has been recently stressed by its relative insensitivity to $C P$ violation parameter values, which may otherwise severely reduce coherent interaction rates $[32,33]$. Nevertheless, $\mathrm{CF}_{3} \mathrm{Br}$ modules able to exploit coherent couplings are presently under development. The intrinsic insensitivity of SDDs to most undesirable backgrounds, low cost of materials involved, and simplicity of production and operation opens a new door to dark matter detection.

We thank the Communauté des Communes du Pays d'Apt and French Ministry of Defense for supporting the conversion of the underground site. Our gratitude goes to M. Auguste, J. Bourges, G. Boyer, R. Brodzinski, A. Cavaillou, COMEX-PRO, M. El-Majd, M. Embid, L. Ibtiouene, IMEC, J. Matricon, M. Minowa, Y. H. Mori, T. Otto, G. Roubaud, M. Same, and C. W. Thomas.
*Corresponding author.

Email address: Juan.Collar@cern.ch

[1] R. E. Apfel, Nucl. Instrum. Methods 162, 603 (1979).

[2] F. Seitz, Phys. Fluids 1, 1 (1958).

[3] S.C. Roy et al., Nucl. Instrum. Methods Phys. Res., Sect. A 255, 199 (1987).

[4] Ch. Peyrou, in Bubble and Spark Chambers, edited by R. P. Shutt (Academic Press, New York, 1967).

[5] R.E. Apfel et al., Phys. Rev. A 31, 3194 (1985).

[6] M. Harper, Ph.D. thesis, University of Maryland, 1991; Nucl. Sci. Eng. 114, 118 (1993); Nucl. Instrum. Methods Phys. Res., Sect. A 336, 220 (1993).

[7] G. Jungman et al., Phys. Rep. 267, 195 (1996).

[8] Proceedings of the 2nd International Workshop on the Identification of Dark Matter, Buxton, 1998 (World Scientific, Singapore, 1999).

[9] J. I. Collar, Phys. Rev. D 54, R1247 (1996).

[10] L. A. Hamel et al., Nucl. Instrum. Methods Phys. Res., Sect. A 388, 91 (1997); N. Boukhira et al., Astropart. Phys. 14, 227 (2000).

[11] V. Zacek, Nuovo Cimento Soc. Ital. Fis. 107A, 291 (1994).

[12] Commercial SDDs based on viscoelastic gels operate continuously for up to 3 mo but allow bubble migration.

[13] J. D. Worley et al., J. Chem. Phys. 45, 2868 (1966).

[14] E. Dendy Sloan, Jr., Clathrate Hydrates of Natural Gases (Marcel Dekker Inc., New York, 1997); Y. H. Mori (private communication).

[15] M. Sugaya and Y.H. Mori, Chem. Eng. Sci. 51, 3505 (1996); Y. H. Mori, Phys. Fluids 8, 2558 (1996); F. Isobe and Y.H. Mori, Rev. Int. Froid 15, 137 (1992).

[16] B. Roy et al., Radiat. Meas. 29, 173 (1998).

[17] R. E. Apfel et al., Rev. Sci. Instrum. 54, 1397 (1983).

[18] Yu. N. Martynyuk and N. S. Smirnova, Sov. Phys. Acoust. 37, 376 (1991); Yu. A. Aleksandrov et al., Bubble Chambers (Indiana University Press, Bloomington, 1967), p. 29.

[19] Y.-Ch. Lo and R. Apfel, Phys. Rev. A 38, 5260 (1988).

[20] M. El-Nagdy et al., J. Br. Nucl. Energy Soc. 10, 131 (1971).

[21] Recent work by F. d'Errico [Radiat. Prot. Dosim. 84, 55 (1999)] simplifies the calculation of $E_{\mathrm{thr}}$.

[22] The ratio of the minimum (Gibbs) expansion work to the sum of surface, vaporization, and expansion energies $[4,6]$.

[23] A. G. Tenner, Nucl. Instrum. Methods 22, 1 (1963).

[24] L. K. Pan et al., Nucl. Instrum. Methods Phys. Res., Sect. A 420, 345 (1999).

[25] "MCNP, A General Monte Carlo N-Particle Transport Code," edited by J. F. Briesmeister, Los Alamos National Laboratory Report No. LA-12625-M, 1993.

[26] L. R. Greenwood and R. K. Smither, "SPECTER, Neutron Damage Calculations for Materials Irradiations," Argonne National Laboratory Report No. ANL/FPP/TM-197, 1985.

[27] http://www.research.ibm.com/ionbeams/

[28] http://home.cern.ch/collar/RUSTREL/rustrel.html; G. Waysand et al., Nucl. Instrum. Methods Phys. Res., Sect. A 444, 336 (2000).

[29] G. Gerbier et al., Astropart. Phys. 11, 287 (1999).

[30] P. F. Smith et al., Phys. Lett. B 379, 299 (1996); J. D. Lewin and P. F. Smith, Astropart. Phys. 6, 87 (1996).

[31] J. Ellis and R. A. Flores, Phys. Lett. B 263, 259 (1991).

[32] T. Falk, A. Ferstl, and K. A. Olive, Phys. Rev. D 59, 055009 (1999); 60, 119904(E) (1999).

[33] J. I. Collar et al., New J. Phys. 2, 14.1 (2000). 